\title{
The status of the TALE surface detector array and a TALE infill project
}

\section{Aoi Iwasaki ${ }^{a}$ and Shoichi Ogio ${ }^{a, b, *}$ on behalf of the Telescope Array Collaboration} (a complete list of authors can be found at the end of the proceedings)

${ }^{a}$ Graduate School of Science, Osaka City University,

3-3-138, Sugimoto, Sumiyoshi-ku, Osaka, Japan

${ }^{b}$ Nambu Yoichiro Institute of Theoretical and Experimental Physics, Osaka City University, 3-3-138, Sugimoto, Sumiyoshi-ku, Osaka, Japan

E-mail: sogio@osaka-cu.ac.jp

Routine hybrid observations of the surface detectors (SD) in conjunction with the fluorescence detectors (FD) of the Telescope Array Low-energy Extension (TALE) began in November 2018. In this presentation, we will describe the simulation studies of detector aperture and resolution of the TALE SD, and report on the latest observation results other than the energy spectrum. We are also in the process of expanding the experiment by 50 SDs, with even smaller nearest-neighbor spacing, in order lower the energy threshold to match that of the Cherenkov-dominated events seen by the FD. Details of the upgrade and expected performance of this new extension will be discussed.

$37^{\text {th }}$ International Cosmic Ray Conference (ICRC 2021)

July 12 th - 23rd, 2021

Online - Berlin, Germany

\footnotetext{
*Presenter
} 


\section{TALE hybrid observations}

The TALE SD array consists of 80 detector units. The spacing of 40 SDs in the distance range of $3 \mathrm{~km}$ from the TALE FD station and the spacing of the other 40 SDs which are located in the range from $3 \mathrm{~km}$ to $5 \mathrm{~km}$ from the TALE-FD station is $600 \mathrm{~m}$. In the connecting area between the TALE SD array and the TA SD array, 23 planned locations for additional SDs are prepared with $1.2 \mathrm{~km}$ spacing for further expansion in the future.

TALE SD holds the same basic design as TA SD based on its satisfactory performance in operation for more than 10 years. Each SD has a plastic scintillation counter of $3 \mathrm{~m}^{2}$ in size, and transmits SD data via a wireless LAN module. Moreover each detector is powered by a solar panel and a battery. The output signals from PMTs are digitized by a 12 bit FADC with a 50 MHz sampling rate on the CPU board. Signal greater than approximately 0.3 MIPs are stored in a memory buffer on the CPU board as Level-0 trigger data. The stored waveform is $2.56 \mu$ s. long (128 FADC bins). Signals greater than 3.0 MIPs are stored as a Level-1 trigger data, which are sent to a single-board computer, called "host PC", which controls trigger decision and data acquisition processes. The local trigger rates are about $750 \mathrm{~Hz}$ for Level- 0 and about $20 \mathrm{~Hz}$ for Level-1. From the Level-1 trigger time tables of all the SDs, an air shower event trigger is generated when any five SDs are coincident with in $\pm 4 \mu \mathrm{s}$. We call this trigger the Level-2 trigger, and the current Level-2 triggering rate is 30 events/min. Routine operation started in September 2019.

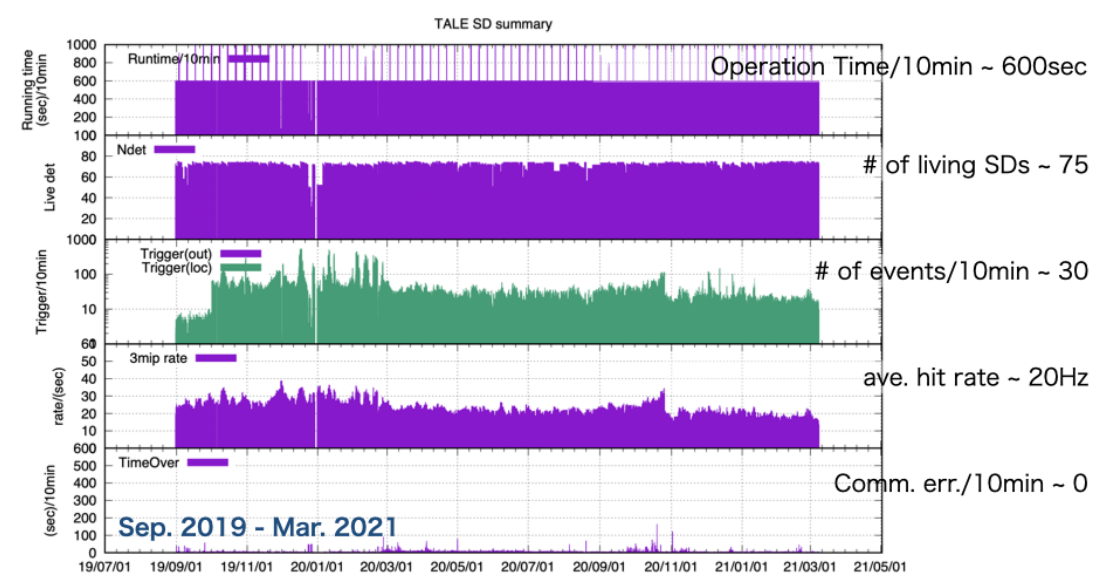

Figure 1: Operation status of the TALE SD array from September 2019.

TALE FD station located at TA Middle Drum (MD) site has ten FD telescopes, which observe $31-59^{\circ}$ in elevation, above the field of view of the original TA FD MD station. The TALE FD station was completed in the fall of 2013 , and has been taking data in monocular mode since that time.

The hybrid trigger condition on the TALE FD DAQ system is very simple, which is the number of hit PMTs $>5$ and the event duration $>500 \mathrm{~ns}$. The hybrid triggering rate is about $0.05 \mathrm{~Hz}$. With the Level-2 or the hybrid trigger trigger the host PC requests each SD to transfer waveform data within $\pm 32 \mu$ s from the trigger timing, and the host PC corrects waveform data. 
The routine hybrid operation started in October 2018, and he number of hybrid events reached 70,000 at February 2020, as shown in Fig. 2 After a suspension due to the COVID-19 pandemic, hybrid operations resumed in December 2020.

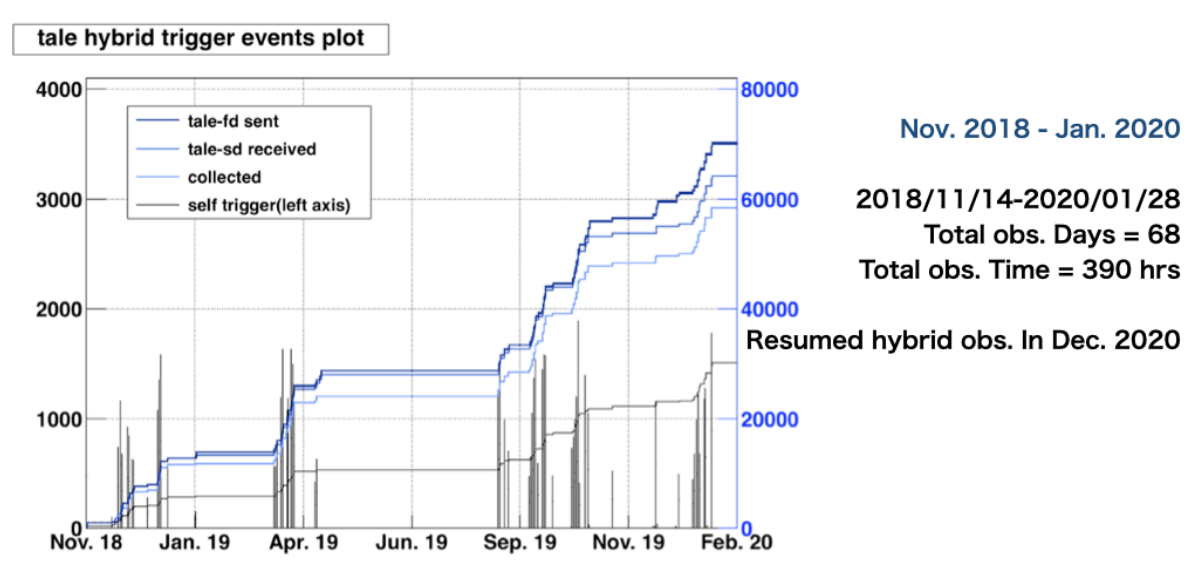

Figure 2: The cumulative number of events with the TALE hybrid trigger.

\section{Extension of TALE hybrid}

Additionally installing TALE infill surface detectors covering very wide energy range. The expected stereo coverage is from $10^{15} \mathrm{eV}$ to $10^{18} \mathrm{eV}$, that is from "knee" to "ankle". We will additionally install 54 SDs with $100 \mathrm{~m}$ and $200 \mathrm{~m}$ spacing near the TALE FD station within $2 \mathrm{~km}$, covering $0.32 \mathrm{~km}^{2}$. Fig 3 shows a layout of the TALE-infill SDs with TALE FD station and TALE SDs.

Fig. 4are expected triggering efficiency of the TALE-infill SD array and of the TALE-infill hybrid. Based on Monte Carlo studies, we expect $10 \%$ efficiency at $10^{* *} \mathrm{eV}$ for the infill SD array and at at $10^{* *} \mathrm{eV}$ for the hybrid trigger operation. From these values, the expected mode energy of the infill SD array is $10^{15.3} \mathrm{eV}$, and for theTALE-infill hybrid it will be reached to $10^{15.8} \mathrm{eV}$.

\section{Acknowledgements}

The TALE SD production and the TALE hybrid operations are supported by the Japan Society for the Promotion of Science(JSPS) through Grants-in-Aid for Scientific Research (S) 15H05741 and 19H05607; by the joint research program of the Institute for Cosmic Ray Research (ICRR), The University of Tokyo. The experimental site became available through the cooperation of the Utah School and Institutional Trust Lands Administration (SITLA), U.S. Bureau of Land Management (BLM), and the U.S. Air Force. We appreciate the assistance of the State of Utah and Fillmore offices of the BLM in crafting the Plan of Development for the site. The people and the officials of Millard County, Utah have been a source of steadfast and warm support for our work which we greatly appreciate. We gratefully acknowledge the contribution from the technical staffs of our home institutions. 


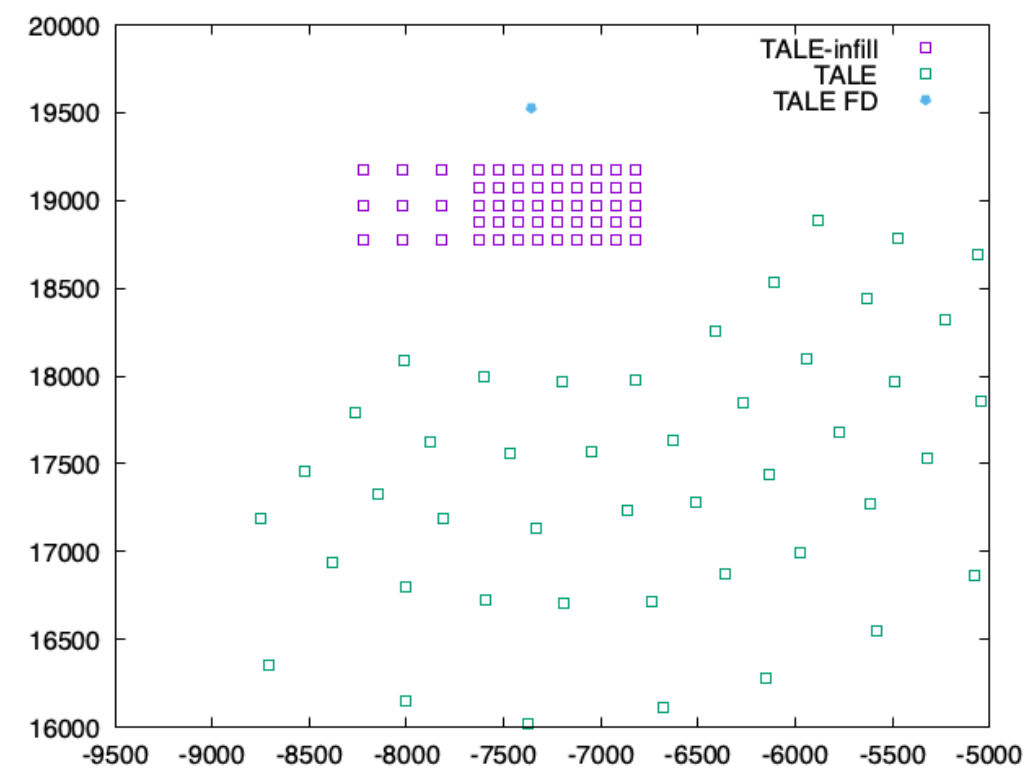

Figure 3: The layout of TALE infill SDs. Purple squares denote the planned locations of 54 SDs for the infill array. The array controlled with a host PC at the TALE FD station indicated with the filled circle.
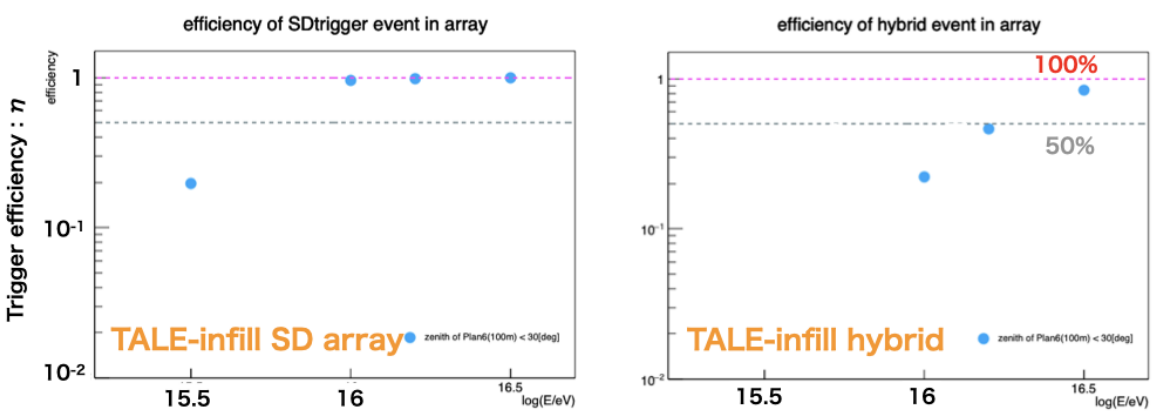

Figure 4: The triggering efficiencies of the TALE-infill SD array and the TALE-infill hybrid operation. 


\section{Full Authors List: Telescope Array Collaboration}

R.U. Abbasi ${ }^{1,2}$, T. Abu-Zayyad ${ }^{1,2}$, M. Allen ${ }^{2}$, Y. Arai $^{3}$, R. Arimura ${ }^{3}$, E. Barcikowski ${ }^{2}$, J.W. Belz ${ }^{2}$, D.R. Bergman ${ }^{2}$, S.A. Blake ${ }^{2}$, I. Buckland ${ }^{2}$, R. Cady $^{2}$, B.G. Cheon ${ }^{4}$, J. Chiba ${ }^{5}$, M. Chikawa ${ }^{6}$, T. Fujii ${ }^{7}$, K. Fujisue ${ }^{6}$, K. Fujita ${ }^{3}$, R. Fujiwara ${ }^{3}$, M. Fukushima ${ }^{6}$, R. Fukushima ${ }^{3}$, G. Furlich ${ }^{2}$, R. Gonzalez ${ }^{2}$, N. Globus ${ }^{13}$, W. Hanlon ${ }^{2}$, M. Hayashi ${ }^{8}$, N. Hayashida ${ }^{9}$, K. Hibino ${ }^{9}$, R. Higuchi ${ }^{6}$, K. Honda ${ }^{10}$, D. Ikeda ${ }^{9}$, T. Inadomi ${ }^{11}$, N. Inoue ${ }^{12}$, T. Ishii ${ }^{10}$, H. Ito $^{13}$, D. Ivanov ${ }^{2}$, H. Iwakura ${ }^{11}$, A. Iwasaki ${ }^{3}$, H.M. Jeong ${ }^{14}$, S. Jeong ${ }^{14}$, C.C.H. Jui ${ }^{2}$, K. Kadota ${ }^{15}$, F. Kakimoto ${ }^{9}$, O. Kalashev ${ }^{16}$, K. Kasahara ${ }^{17}$, S. Kasami ${ }^{18}$, H. Kawai ${ }^{19}$, S. Kawakami ${ }^{3}$, S. Kawana ${ }^{12}$, K. Kawata ${ }^{6}$, I. Kharuk ${ }^{16}$, E. Kido ${ }^{13}$, H.B. Kim ${ }^{4}$, J.H. Kim ${ }^{2}$, J.H. Kim² ${ }^{2}$ M.H. Kim ${ }^{14}$, S.W. Kim ${ }^{14}$, Y. Kimura ${ }^{3}$, S. Kishigami ${ }^{3}$, Y. Kubota ${ }^{11}$, S. Kurisu ${ }^{11}$, V. Kuzmin ${ }^{16}$, M. Kuznetsov ${ }^{16,20}$, Y.J. Kwon ${ }^{21}$, K.H. Lee ${ }^{14}$, B. Lubsandorzhiev ${ }^{16}$, J.P. Lundquist ${ }^{2,22}$, K. Machida ${ }^{10}$, H. Matsumiya ${ }^{3}$, T. Matsuyama ${ }^{3}$, J.N. Matthews ${ }^{2}$, R. Mayta ${ }^{3}$, M. Minamino ${ }^{3}$, K. Mukai ${ }^{10}$, I. Myers $^{2}$, S. Nagataki ${ }^{13}$, K. Nakai ${ }^{3}$, R. Nakamura ${ }^{11}$, T. Nakamura ${ }^{23}$, T. Nakamura ${ }^{11}$, Y. Nakamura ${ }^{11}$, A. Nakazawa ${ }^{11}$, E. Nishio ${ }^{18}$, T. Nonaka ${ }^{6}$, H. Oda ${ }^{3}$, S. Ogio ${ }^{3,24}$, M. Ohnishi ${ }^{6}$, H. Ohoka ${ }^{6}$, Y. Oku ${ }^{18}$, T. Okuda ${ }^{25}$, Y. Omura ${ }^{3}$, M. Ono ${ }^{13}$, R. Onogi ${ }^{3}$, A. Oshima ${ }^{3}$, S. Ozawa ${ }^{26}$, I.H. Park ${ }^{14}$, M. Potts ${ }^{2}$, M.S. Pshirkov ${ }^{16,27}$, J. Remington ${ }^{2}$, D.C. Rodriguez ${ }^{2}$, G.I. Rubtsov ${ }^{16}$, D. Ryu ${ }^{28}$, H. Sagawa ${ }^{6}$, R. Sahara ${ }^{3}$, Y. Saito ${ }^{11}$, N. Sakaki ${ }^{6}$, T. Sako ${ }^{6}$, N. Sakurai ${ }^{3}$, K. Sano ${ }^{11}$, K. Sato ${ }^{3}$, T. Seki ${ }^{11}$, K. Sekino ${ }^{6}$, P.D. Shah ${ }^{2}$, Y. Shibasaki ${ }^{11}$, F. Shibata ${ }^{10}$, N. Shibata ${ }^{18}$, T. Shibata ${ }^{6}$, H. Shimodaira ${ }^{6}$, B.K. Shin ${ }^{28}$, H.S. Shin ${ }^{6}$, D. Shinto ${ }^{18}$, J.D. Smith ${ }^{2}$, P. Sokolsky ${ }^{2}$, N. Sone ${ }^{11}$, B.T. Stokes ${ }^{2}$, T.A. Stroman ${ }^{2}$,

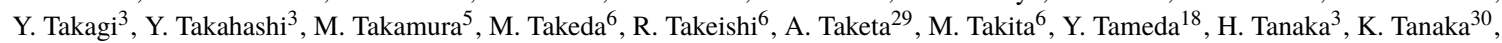
M. Tanaka ${ }^{31}$, Y. Tanoue ${ }^{3}$, S.B. Thomas ${ }^{2}$, G.B. Thomson ${ }^{2}$, P. Tinyakov ${ }^{16,20}$, I. Tkachev ${ }^{16}$, H. Tokuno ${ }^{32}$, T. Tomida ${ }^{11}$, S. Troitsky ${ }^{16}$,

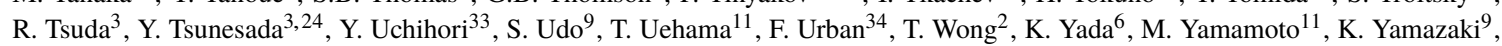
J. Yang ${ }^{35}$, K. Yashiro ${ }^{5}$, F. Yoshida ${ }^{18}$, Y. Yoshioka ${ }^{11}$, Y. Zhezher ${ }^{6,16}$, and Z. Zundel ${ }^{2}$

${ }^{1}$ Department of Physics, Loyola University Chicago, Chicago, Illinois, USA

${ }^{2}$ High Energy Astrophysics Institute and Department of Physics and Astronomy, University of Utah, Salt Lake City, Utah, USA

${ }^{3}$ Graduate School of Science, Osaka City University, Osaka, Osaka, Japan

${ }^{4}$ Department of Physics and The Research Institute of Natural Science, Hanyang University, Seongdong-gu, Seoul, Korea

${ }^{5}$ Department of Physics, Tokyo University of Science, Noda, Chiba, Japan

${ }^{6}$ Institute for Cosmic Ray Research, University of Tokyo, Kashiwa, Chiba, Japan

${ }^{7}$ The Hakubi Center for Advanced Research and Graduate School of Science, Kyoto University, Kitashirakawa-Oiwakecho, Sakyo-ku, Kyoto, Japan

${ }^{8}$ Information Engineering Graduate School of Science and Technology, Shinshu University, Nagano, Nagano, Japan

${ }^{9}$ Faculty of Engineering, Kanagawa University, Yokohama, Kanagawa, Japan

${ }^{10}$ Interdisciplinary Graduate School of Medicine and Engineering, University of Yamanashi, Kofu, Yamanashi, Japan

${ }^{11}$ Academic Assembly School of Science and Technology Institute of Engineering, Shinshu University, Nagano, Nagano, Japan

12 The Graduate School of Science and Engineering, Saitama University, Saitama, Saitama, Japan

${ }^{13}$ Astrophysical Big Bang Laboratory, RIKEN, Wako, Saitama, Japan

${ }^{14}$ Department of Physics, SungKyunKwan University, Jang-an-gu, Suwon, Korea

${ }^{15}$ Department of Physics, Tokyo City University, Setagaya-ku, Tokyo, Japan

${ }^{16}$ Institute for Nuclear Research of the Russian Academy of Sciences, Moscow, Russia

${ }^{17}$ Faculty of Systems Engineering and Science, Shibaura Institute of Technology, Minato-ku, Tokyo, Japan

${ }^{18}$ Department of Engineering Science, Faculty of Engineering, Osaka Electro-Communication University, Neyagawa-shi, Osaka, Japan

${ }^{19}$ Department of Physics, Chiba University, Chiba, Chiba, Japan

${ }^{20}$ Service de Physique Theorique, Universite Libre de Bruxelles, Brussels, Belgium

${ }^{21}$ Department of Physics, Yonsei University, Seodaemun-gu, Seoul, Korea

${ }^{22}$ Center for Astrophysics and Cosmology, University of Nova Gorica, Nova Gorica, Slovenia

${ }^{23}$ Faculty of Science, Kochi University, Kochi, Kochi, Japan

${ }^{24}$ Nambu Yoichiro Institute of Theoretical and Experimental Physics, Osaka City University, Osaka, Osaka, Japan

${ }^{25}$ Department of Physical Sciences, Ritsumeikan University, Kusatsu, Shiga, Japan

${ }^{26}$ Quantum ICT Advanced Development Center, National Institute for Information and Communications Technology, Koganei, Tokyo, Japan

${ }^{27}$ Sternberg Astronomical Institute, Moscow M.V. Lomonosov State University, Moscow, Russia

${ }^{28}$ Department of Physics, School of Natural Sciences, Ulsan National Institute of Science and Technology, UNIST-gil, Ulsan, Korea

${ }^{29}$ Earthquake Research Institute, University of Tokyo, Bunkyo-ku, Tokyo, Japan

${ }^{30}$ Graduate School of Information Sciences, Hiroshima City University, Hiroshima, Hiroshima, Japan

${ }^{31}$ Institute of Particle and Nuclear Studies, KEK, Tsukuba, Ibaraki, Japan

${ }^{32}$ Graduate School of Science and Engineering, Tokyo Institute of Technology, Meguro, Tokyo, Japan

33 Department of Research Planning and Promotion, Quantum Medical Science Directorate, National Institutes for Quantum and Radiological Science and Technology, Chiba, Chiba, Japan

${ }^{34}$ CEICO, Institute of Physics, Czech Academy of Sciences, Prague, Czech Republic

${ }^{35}$ Department of Physics and Institute for the Early Universe, Ewha Womans University, Seodaaemun-gu, Seoul, Korea 